Journal of Applied Mathematics and Simulation Vol. 1, Issue 1, 1987.

TOPOLOGICAL STRUCTURES IN COMPUTER SCIENCE

\author{
EFIM KHALIMSKY \\ City University of New York \\ CSI \\ Staten Island, NY 10301
}

\begin{abstract}
Topologies of finite spaces and spaces with countably many points are investigated. It is proven, using the theory of ordered topological spaces, that any topology in connected ordered spaces, with finitely many points or in spaces similar to the set of all integers, is an interval-alternating topology. Integer and digital lines, arcs, and curves are considered. Topology of $\mathrm{N}$-dimensional digital spaces is described. A digital analog of the intermediate value theorem is proven. The equivalence of connectedness and pathconnectedness in digital and integer spaces is also proven. It is shown here how methods of continuous mathematics, for example, topological methods, can be applied to objects, that used to be investigated only by methods of discrete mathematics. The significance of methods and ideas in digital image and picture processing, robotic vision, computer tomography and system's sciences presented here is well known.

Key words: ordered topological spaces, finite topological spaces, $\mathrm{T}_{\mathrm{o}^{-}}$ topology, product topology, interval-alternating topology, connectedness, pathconnectedness, digital arcs, lines and curves, quantized spaces, cross-section of ordered set, digital analog of intermediate value theorem, digital picture processing, robot vision, computer tomography, discrete mathematics.
\end{abstract}

AMS subject classification: 54F05

\title{
INTRODUCTION
}

Interior, closure, boundary of a set, domain, topological product, connectedness, arcs, curves, surfaces, fundamental groups, homotopy and homology groups are some fundamental terms, concepts and ideas which one can find in any textbook on topology (see, for example, [1-5]). These and many other topological concepts play a significant role in computer areas such as: digital image processing, picture processing, computer graphics, computer tomography, computer and robot vision, computer networks. Pixels (squares), voxels (cubes) and numbers, which are finite sequences with finitely many symbols (digits, i.e., $n$ digits in n-ary numerical system), are elementary objects that play the role of points in topology. In any case, there are always finitely many objects. But the only 
possible $T_{1}$ or Hausdorff topology in finite sets is the discrete topology, under which all arcs, curves, paths and components are trivial. So, it does not make any sense to use such a topology for those sets. On the other hand, non-Hausdorff and non- $\mathrm{T}_{1}$ topologies are almost forgotten. Most basic definitions, for instance, of arcs, curves, pathconnectedness, compactness, and many others, include the Hausdorff or $\mathrm{T}_{1}$ separation axiom. Consequently, researchers who applied topology to computer sciences, could hardly operate with these concepts except for arcs, curves, parts, connectedness, and neighborhoods. Moreover, they lost their topological nature and became combinatorial. For example, the concepts of 4-neighborhoods and 8-neighborhoods in digital pictures are given with no definition of a topology [6-7]. Definitions of 4 (8)-arcs, 4 (8)-curves were useful in digital pictures to obtain many important results [6-9]. But their application is limited because of their nontopological nature. They can not be used for higher dimensional digital spaces. Furthermore, some results are obtained in a similar way for 3-dimensional digital spaces [10].

Many authors recognized problems arising from combinatorial methods to such notions, as connectivity in digital (or, as they called them, quantized) spaces and tried to solve them in different ways [11-13]. Others tried to find some algebraic, meaningful counterparts of topological notions for such spaces [14-15].

Here we use a strictly topological approach to digital spaces with finitely many points. Definitions of ordered topological spaces (called segments), given below, can be used for sets with finitely many points, as well as with infinitely many points. Some properties of such spaces are considered in the author's earlier publications [16-18], and in his book [19]. N-dimensional and Hilbert spaces are considered as topological products of ordered spaces. Connectedness in such spaces is understood in the usual sense. Arcs, curves, and paths are considered as images of segments which are similar to their definitions as continuous images of the segment $[0,1]$ in topology. It is proven below, that the topologies in n-dimensional digital spaces considered are the only ones possible. Some of the results on digital image processing and digital pattern recognition were presented by the author in [20-23].

In order to make this paper self-contained, a brief review of some important concepts and results from [20] is given without proofs.

Ordered sets, mappings and segments will be understood as linearly ordered sets, continuous mappings, and connected ordered topological spaces, respectively.

Definition 1. A connected space A is said to be a connected ordered topological space or a segment, if it satisfies the following conditions:

a) There are no more than two points $\mathrm{a}_{1}, \mathrm{a}_{2}$ (called end points) in $\mathrm{A}$, such that $\mathrm{A}-\left\{\mathrm{a}_{\mathrm{i}}\right\}$ $(i=1,2)$ is a connected subspace of $A$;

b) For every point $\mathrm{a} \in \mathrm{A}$ different from end points, the space $\mathrm{A}-\{\mathrm{a}\}$ contains exactly two components;

c) If a component $U_{a}$ of the space $A-\{a\}$ contains either a connected component $U_{b}$ of the space $A-\{b\}$ or an end point $a_{i}$ of the segment $A$, then the connected component $U_{c}$ of the space $A-\{c\}$, which also contains either $U_{b}$ or $a_{i}$, respectively, is either contained in $U_{a}$, or contains $U_{a}(a, b, c \in A)$. 


\section{Theorem 1.}

I. If a point $\mathrm{a}$ is not an end point of a segment $\mathrm{A}$, then every component of $\mathrm{A}-\{\mathrm{a}\}$ is either an open, but is not a closed subset of A, or it is a closed, but is not an open subset of A.

II. If a component of $\mathrm{A}-\{\mathrm{a}\}$ is open (closed) subset of $\mathrm{A}$, then another one is also open (closed) subset of $\mathrm{A}$.

III. If a point $\mathrm{a}$ is not an end point of $\mathrm{A}$, then the following statements are equivalent:

1) $U_{a}$ is an open (closed) subset of $A$;

2) $U_{a} \cup\{a\}$ is a closed (open) subset of $A$;

3) $\{\mathrm{a}\}$ is a closed (open) subset of $\mathrm{A}$. If $\mathrm{a}$ is an end point of $\mathrm{A}$, then statements 1 and 3 are equivalent.

Corollary 1. If a is not an end point of a segment $\mathrm{A}$, then a is either an open, or a closed subset of A.

Theorem 2. Let $\mathrm{a}_{1}, \mathrm{a}_{2}$ be arbitrary points of a segment $\mathrm{A}$. If $\mathrm{a}_{1} \neq \mathrm{a}_{2}$, then the component of $A-\left\{a_{2}\right\}$, which does not contain $a_{1}$, is a subset of the component of $A-\left\{a_{1}\right\}$, containing $a_{2}$, and the other component of $A-\left\{a_{1}\right\}$ is a subset of the other component of $A-\left\{a_{2}\right\}$. If $a_{1} \neq a_{2}$, and a component of $A-\left\{a_{2}\right\}$ is a subset of some component $U_{1}$ of $A-\left\{a_{1}\right\}$, then $a_{2} \in U_{1}$.

Definition 2. Two sets are said to be comparable if one of them is a subset of the other one, otherwise, they are noncomparable.

Corollary 2. For a pair of points $\mathrm{a}, \mathrm{a}_{1} \in \mathrm{A}$ and for a component $\mathrm{U}_{\mathrm{a}}$ of $\mathrm{A}-\{\mathrm{a}\}$, there is no comparable component of $A-\left\{a_{1}\right\}$ iff $a_{1}$ is an end point of $A$ and $a_{1} \in U_{a}$.

Definition 3. Let $\mathrm{R}$ be a set of all subsets of a segment $A$, each one of which is either a component of $\mathrm{A}-\left\{\mathrm{a}_{1}\right\}$ for some $\mathrm{a}_{1} \in \mathrm{A}$, or it is an end point of $\mathrm{A}$. Set $\mathrm{R}$ is broken into two equivalence classes in such a way, that every two elements of one class are comparable. Let $R_{1}$ be one of the classes, and let $R_{2}$ be another one. Elements of $R_{1}\left(R_{2}\right)$ are called $\mathrm{U}_{\mathrm{a}}\left(\mathrm{U}_{\mathrm{a}}^{\prime}\right)$. Let us order the set of all points of the segment $\mathrm{A}$. We use the term

"equal" only for coinciding elements of $A$. We call $a_{1}<a_{2} \in U_{a_{2}}$.

If another equivalence class is called $\mathrm{R}_{1}$, then we get an order on $\mathrm{A}$ opposite to the order we got before. Two orders on A are said to be orders induced by connectedness of a segment.

Definition 4. An oriented segment is said to be a segment with the order induced by its connectedness. A segment different from segment $\overrightarrow{\mathrm{A}}$ only by its orientation is called $\stackrel{\mathrm{A}}{\text {. }}$ 
Theorem 3. For every pair of points $a_{1}, a_{2} \in \vec{A}$ exactly one of the relations $a_{1}<a_{2}, a_{1}>$ $\mathrm{a}_{2}, \mathrm{a}_{1}=\mathrm{a}_{2}$ is true.

Corollary 3. If $\mathrm{a} \in \overrightarrow{\mathrm{A}}, \mathrm{a}_{1} \in \mathrm{U}_{\mathrm{a}}$ and $\mathrm{a}_{2} \in \mathrm{U}_{\mathrm{a}}^{\prime}$, then $\mathrm{a}_{1}<\mathrm{a}<\mathrm{a}_{2}$.

Corollary 4. If $a \in \vec{A}$ is not an end point of a segment $\vec{A}$, then $\left.U_{a}=\right] \leftarrow, a\left[; U_{a}^{-}=\right.$ ] a, $\left.\rightarrow\left[; U_{a} \cup\{a\}=\right] \leftarrow, a\right] ; U_{a} \cup\{a\}=[a, \rightarrow[$. If $a \in \vec{A}$ is an end point of segment $\vec{A}$, then $\left.\vec{A}-\{a\}=U_{a}^{-}=\right] a, \rightarrow\left[\right.$ if $\{a\} \in R_{1}$, and $\left.\vec{A}-\{a\}=U_{a}=\right] \leftarrow$, a $\left[\right.$, if $a \in R_{2}$.

Corollary 5. If a is not an end point of segment $\overrightarrow{\mathrm{A}}$, then:

a) Each of the sets ] $\leftarrow$, a [ ; ] a, $\rightarrow$ [ is either an only open, or an only closed subset of segment $\overrightarrow{\mathrm{A}}$;

b) If one of the sets is open (closed) in $\vec{A}$, then the other one is also open (closed) in $\vec{A}$;

c) If $\mathrm{a}$ is not an end point of $\overrightarrow{\mathrm{A}}$, then the following statements are equivalent:

1) Set ] $\leftarrow$, $[$ is open (closed) in $\vec{A}$;

2) Set ] $\leftarrow$, a ] is closed (open) in $\vec{A}$;

3) Set $\{a\}$ is closed (open) in $\vec{A}$;

If $\mathrm{a}$ is an end point of segment $\overrightarrow{\mathrm{A}}$, then statements 1) and 3) are equivalent.

Definition 5. Arcs are homeomorphic images of connected ordered topological spaces; paths are continuous images; curves are qutient spaces of segments with two end points, obtained by their identification, whenever they both are either open, or closed subsets of considered segments.

Definition 6. By an order topology (O-topology) in an ordered set $\mathrm{C}$ we understand a topology in $\mathrm{C}$ such, that $\mathrm{C}$ is a segment, in which one of the orderings, induced by its connectedness, coincides with the given ordering.

Lemma 1. Let ( A, B ) be a cross-section in an ordered set C such, that A (B) does not contain the maximum (minimum) element. In order that the topology in $\mathrm{C}$ be an O-topology it is necessary that A (B) be an open set under this topology.

Proof. For every $\mathrm{a}_{1} \in \mathrm{A}$, there is $\mathrm{a}_{2} \in \mathrm{A}$ such, that $\mathrm{a}_{1}<\mathrm{a}_{2}$. One of the sets ] $\leftarrow \mathrm{a}_{2}[$ or ]$\left.\leftarrow, a_{2}\right]$ is open, by corollary 5 . This proves, that for every element $a_{1} \in A$ there is an open neighborhood of $a_{1}$ in A. So A is an open subset of C. Similar result for B could be obtained by changing the order on $\mathrm{C}$ for the opposite one.

Theorem 4. If an ordered set $\mathrm{C}$ contains gaps, than $\mathrm{C}$ does not admit any O-topology. 
Proof. Let a cross-section (A,B) in ordered set $\mathrm{C}$ be a gap. Assuming that $\mathrm{C}$ admits an $\mathrm{O}$ topology $\mathrm{T}$ we get a contradiction as follows: by lemma 1, each one of the sets $\mathrm{A}$ and $\mathrm{B}$ is an open subset of segment $C$ with topology $T$, and, by definition of a segment, $C$ is connected and so, it cannot be represented as a union of two nontrivial open subsets $\mathrm{A} \cup \mathrm{B}$.

Corollary 6. No one cross-section in an ordered set of points of a segment, with order induced by its connectedness, is a gap.

Theorem 5. If for a point $\mathrm{a} \in \overrightarrow{\mathrm{C}}$, each set of the type ] b, a [ ( ] a, b [ ), where $\mathrm{b}<\mathrm{a}(\mathrm{a}<\mathrm{b})$ is infinite, then $\{a\}$ is a closed set in $\overrightarrow{\mathrm{C}}$.

Proof. Let ( A, B ) be a cross-section of a given ordered set $\mathrm{C}$ such that $\mathrm{A}=] \leftarrow$, a $[$; $\mathrm{B}=$ $[\mathrm{a}, \rightarrow[(\mathrm{A}=] \leftarrow, \mathrm{a}] ; \mathrm{B}=] \mathrm{a}, \rightarrow[\mathrm{)})$. By lemma $1, \mathrm{~A}(\mathrm{~B})$ is an open subset of $\mathrm{C}$, so, by corollary $5,\{a\}$ is a closed set in $\vec{C}$.

Definition $6^{\prime}$. Let $\mathrm{C}$ is an ordered set. Element $\mathrm{a} \in \overrightarrow{\mathrm{C}}$ is a limit element of an ordered set $\mathrm{C}$, iff at least one of the following conditions is true:

1) for every element $b \in C$, such that $b<a$, the set ] b, a [ is nonempty;

2) for every element $b \in C$, such that $a<b$, the set ] $a, b[$ is nonempty.

Element $\mathrm{a} \in \mathrm{C}$ is an isolated element of an ordered set $\mathrm{C}_{1}$ iff it is not a limit element of $\mathrm{C}$. So, by theorem 5 , if $a \in \vec{C}$ is a limit element of $\vec{C}$, then a is a closed subset of $\vec{C}$.

Theorem 6. If a is an end-point of a segment $\overrightarrow{\mathrm{C}}$, different from an antidiscrete two points set, then $\{\mathrm{a}\}$ - is either only an open set in $\overrightarrow{\mathrm{C}}$, or - only a closed set in $\overrightarrow{\mathrm{C}}$.

Proof: If a is a limit element of an ordered set $\mathrm{C}$, then, by theorem 5, $\{\mathrm{a}\}$ is a closed subset of $\overrightarrow{\mathrm{C}}$. If a is an isolated element of an ordered set $\mathrm{C}$, then one of the following conditions holds:

1) if a is the first element of an ordered set $\vec{C}$, then there is an element $b \in C$ such, that $\mathrm{a}<\mathrm{b}$, and the set $] \mathrm{a}, \mathrm{b}[=\varnothing$;

2) if a is the last element of $\overrightarrow{\mathrm{C}}$, then there is an element $\mathrm{b} \in \overrightarrow{\mathrm{C}}$ such, that $\mathrm{b}<\mathrm{a}$ and ] b, a $[=\varnothing$. Proof.

If $\{\vec{C}\}=\{a, b\}$, then all logically possible topologies on $C$ are following:

1) $\varnothing,\{a, b\}$ - are open;

2) $\varnothing,\{a, b\},\{a\}$ - are open;

3) $\varnothing\{a, b\},\{b\}$ - are open;

4) $\varnothing,\{a, b\},\{a\},\{b\}-$ are open. 
If $\{\vec{C}\} \neq\{a, b\}$, and $a$ is an isolated element of $\vec{C}$, then $b$ is not an end point of $\vec{C}$. By corollary $1,\{b\}$ is either an open, or a closed subset of $\vec{C} \cdot U_{b}=\{a\}\left(U_{b}=a\right)$. By theorem $1, U_{b}\left(U_{b}\right)$, i.e. $\{a\}$ is either a closed, or an open subset of $\vec{C}$.

Corollary 7. If the segment $\overrightarrow{\mathrm{C}}$ is not an antidiscrete two points set, and $\mathrm{a} \in \overrightarrow{\mathrm{C}}$, then $\{\mathrm{a}\}$ is either an open, or a closed subset of $\overrightarrow{\mathrm{C}}$.

Proof follows immediately from corollary 1 and theorem 6 .

Theorem 7. If there exists a decomposition of an ordered set $\mathrm{C}$ into three non-empty subsets $A_{1}, A_{2}, A_{3}$, such that $a_{1}<a_{2}<a_{3}\left(a_{1}, a_{2}, a_{3}\right.$ are arbitrary elements of the sets $A_{1}$, $A_{2}, A_{3}$, respectively), and $A_{1}$ does not contain a maximal element, $A_{3}$ does not contain a minimal element, and $\mathrm{A}_{2}$ consists of finite even number of elements, then $\mathrm{C}$ does not admit any O-topology.

Proof: Subset $\mathrm{A}_{2}$ of ordered set $\mathrm{C}$ is a sequence of type $\mathrm{a}_{1}<\mathrm{a}_{2}<\ldots<\mathrm{a}_{2 \mathrm{~K}}$, where $\mathrm{k}$ is a natural number, and $] \mathrm{a}_{\mathrm{i}}, \mathrm{a}_{\mathrm{i}+1}[=\varnothing(\mathrm{i}=1,2, \ldots, 2 \mathrm{k}-1)$. Let us assume, that there is an O-topology $\mathrm{T}$ on $\mathrm{C}$, such that ordered set $\mathrm{C}$ with topology $\mathrm{T}$ is a segment of $\overrightarrow{\mathrm{C}}$. Points $\mathrm{a}_{1}$, $a_{2 k}$ are limit points of an ordered set $C$ and, by theorem $5,\left\{a_{1}\right\},\left\{a_{2 k}\right\}$ are closed subsets of $\overrightarrow{\mathrm{C}}$. From corollary 5 , it follows, that $\left.] \leftarrow, \mathrm{a}_{1}\right]$ is a closed subset of $\overrightarrow{\mathrm{C}}$. Because ]$\left.\leftarrow, a_{2}[=] \leftarrow, a_{1}\right]$, the set $] \leftarrow, a_{2}\left[\right.$ is a closed subset of $\vec{C}$. By corollary $5,\left\{a_{2}\right\}$ is an open subset of $\overrightarrow{\mathrm{C}}$. Using the mathematical induction we get, that every set of type $\left\{\mathrm{a}_{2 n-1}\right\}$ is a closed subset of $\overrightarrow{\mathrm{C}}$, and every set of type $\left\{\mathrm{a}_{2 n}\right\}$, where $1 \leq n \leq k$, is an open subset of $\overrightarrow{\mathrm{C}}$. So, we got a clopen subset $\left\{\mathrm{a}_{2 \mathrm{k}}\right\}$ of $\overrightarrow{\mathrm{C}}$. This contradicts the connectedness of segment $\overrightarrow{\mathrm{C}}$, and proves that there is no O-topology on $\mathrm{C}$.

Definition 7. An element a of a subset $\mathrm{U}$ of an ordered set $\mathrm{C}$ will be called an end-element of $U$ in $C$, provided there exist an interval [ $a_{1}, a\left[\right.$ or ] $a, a_{1}$ ], whose intersection with $U$ is empty.

Theorem 8. If a is an end-element of an open (closed) set $U$ of the segment $\overrightarrow{\mathrm{C}}$, then the set $\{\mathrm{a}\}$ is open (closed) in $\overrightarrow{\mathrm{C}}$.

Proof: Let, for example, $\left[\mathrm{a}_{1}, \mathrm{a}[\cap \mathrm{U}=\varnothing\right.$. If $\overrightarrow{\mathrm{C}}$ is not an antidiscrete two points set and a $\in \overrightarrow{\mathrm{C}}$ then, by corollary $7,\{\mathrm{a}\}$ is either an open, but not a closed subset of $\overrightarrow{\mathrm{C}}$, or a closed, but not an open subset of $\vec{C}$. So, assuming opposite to what is to be proven, we get, that the end element a of an open (closed) set $U$ in $C$ is a closed (open) subset of $\vec{C}$. By corollary $5,[\mathrm{a}, \rightarrow[$ is closed (open) and ] a, $\rightarrow[$ is open (closed) subset of $\overrightarrow{\mathrm{C}}$, also one of two sets $] \mathrm{a}_{1}, \rightarrow\left[\right.$ or $\left[\mathrm{a}_{1}, \rightarrow[\right.$ is an open (closed) subset of $\overrightarrow{\mathrm{C}}$. Without loss of generality, we can assume, that $\left[a_{1}, \rightarrow[\right.$ is an open (closed) subset of $\vec{C}$. It follows from equality $\left[\mathrm{a}, \rightarrow[=] \mathrm{a}, \rightarrow\left[\cup\left(\mathrm{U} \cap\left[\mathrm{a}_{1}, \rightarrow[\right.\right.\right.\right.$ ), that $[\mathrm{a}, \rightarrow[$ is an open (closed) subset of $\overrightarrow{\mathrm{C}}$. Together with previous, it yields that $[\mathrm{a}, \rightarrow[$ is a clopen subset of $\mathrm{C}$; this contradicts the 
connectedness of $\overrightarrow{\mathrm{C}}$. A similar contradiction will be obtained in the case, when ] $\mathrm{a}_{1}, \rightarrow[$ is an open (closed) subset of $\overrightarrow{\mathrm{C}}$, from the following equality $[\mathrm{a}, \rightarrow[=] \mathrm{a}, \rightarrow[\mathrm{U}$ (Uก $\left[a_{1}, \rightarrow[\right.$ ). In the case, when $] a, a_{1}[\cap U=\varnothing$, the prove could be obtained by replacing the order in $\overrightarrow{\mathrm{C}}$ with the opposite.

Corollary 9. If $\{a\}$ is a closed (open) subset of $\overrightarrow{\mathrm{C}}$, and there is a point $\mathrm{a}_{1} \in \overrightarrow{\mathrm{C}}$ such, that either $] \mathrm{a}_{1}, \mathrm{a}[=\varnothing$, or $] \mathrm{a}, \mathrm{a}_{1}[=\varnothing$, then each open (closed) subset $\mathrm{U} \subset \mathrm{C}$, which contains a, contains also $\mathrm{a}_{1}$.

Proof. If there is an open (closed) set $U$ in $\vec{C}$, which contains a, but does not contain $a_{1}$, then $\mathrm{a}$ is an end-element of $\mathrm{U}$ and, consequently, by theorem $8,\{\mathrm{a}\}$ is an open (closed) in $\overrightarrow{\mathrm{C}}$. Hence, $\{\mathrm{a}\}$ is a clopen subset of $\overrightarrow{\mathrm{C}}$, what contradicts the connectedness of $\overrightarrow{\mathrm{C}}$.

Corollary 10. If a point a is not an end point of the segment $\vec{C}$ and $\{a\}$ is an open set in $\vec{C}$, then there exist points $a_{1}$ and $a_{2}$ in $\vec{C}$, such that $] a_{1}, a_{2}[=\{a\}$; in such a case the closure of $\{a\}$ is equal to [ $\left.a_{1}, a_{2}\right]$. If $a$ is the first (the last) element of $\vec{C}$ and $\{a\}$ is an open set in $\overrightarrow{\mathrm{C}}$, then there exists a point $\mathrm{a}_{1}$ in $\overrightarrow{\mathrm{C}}$, such that $] \leftarrow, \mathrm{a}_{1}\left[=\{\mathrm{a}\}(] \mathrm{a}_{1}, \rightarrow[=\{\mathrm{a}\})\right.$; thus the closure of $\{a\}$ is equal to $\left[a, a_{1}\right]\left(\left[a_{1}, a\right]\right)$ (the existance of both points $a_{1}, a_{2}$ follows from theorem 5).

Proof. Let a is not an end point of $\overrightarrow{\mathrm{C}}$. Because $\{\mathrm{a}\}$ is an open set in $\overrightarrow{\mathrm{C}}$, it follows, that each one of the sets $U_{a}, U_{a}^{\prime}$ is a closed set in $\vec{C}$. From equalities $U_{a}=U_{a_{1}} \cup\left\{a_{1}\right\}$ and $U_{a}^{\prime}=$ $U_{a_{2}}^{\prime} \cup\left\{a_{2}\right\}$ it follows, that sets $\left\{a_{1}\right\}$ and $\left\{a_{2}\right\}$ are closed in $\vec{C}$. Hence, each one of the sets $\mathrm{U}_{\mathrm{a}_{1}}^{\prime} \cup\left\{\mathrm{a}_{1}\right\}$ and $\mathrm{U}_{\mathrm{a}_{2}} \cup\left\{\mathrm{a}_{2}\right\}$ is closed in $\overrightarrow{\mathrm{C}}$; set $\left[\mathrm{a}_{1}, \mathrm{a}_{2}\right]$ is a closed subset of $\overrightarrow{\mathrm{C}}$, as an intersection of those two sets. So, $\left[a_{1}, a_{2}\right] \supset C 1\{a\}$ and, by theorem $8,\left[a_{1}, a_{2}\right] \subset C 1$ $\{a\}$. Hence, $\mathrm{C} 1\{\mathrm{a}\}=\left[\mathrm{a}_{1}, \mathrm{a}_{2}\right]$. If $\mathrm{a}$ is the first (the last) element of $\overrightarrow{\mathrm{C}}$, then the proof is similar to that given above.

Theorem 9. A connected space $\mathrm{A}$ is a segment $\overrightarrow{\mathrm{A}}$, iff its points can be ordered so that only subsets are connected, which along with any two points $\mathrm{a}$ and $\mathrm{b}$, such that $\mathrm{a}<\mathrm{b}$, contain all the points of $\mathrm{A}$, lying between them, i.e. contain [ $\mathrm{a}, \mathrm{b}]$.

\section{Proof:}

1) First, we prove, that a space $\mathrm{A}$ with a fixed order $\mathrm{O}$, satisfying all the conditions of this theorem, is a segment. For every point $a \in A$, which is neither the first, nor the last point of $\mathrm{A}$, the set $\mathrm{A}-\{\mathrm{a}\}$ is disconnected and consists of two components ]$\leftarrow$, $\mathrm{a}$ [ and ] $\mathrm{a}, \rightarrow[$. In the case, when $\mathrm{a}$ is the first (last) element of the ordered set $A$, the set $A-\{a\}=] a, \rightarrow[(A-\{a\}=] \leftarrow, a[)$ is connected. Space $A$ is also connected. If a component $] \leftarrow, \mathrm{a}_{1}\left[\right.$ of $\mathrm{A}-\left\{\mathrm{a}_{1}\right\}$ contains a component ] $\leftarrow, \mathrm{a}_{2}$ [ of $\mathrm{A}$ 
- $\left\{a_{2}\right\}$ or the first element $a_{0}$ of $A$, then a component $] \leftarrow, a_{3}\left[\right.$ of $A-\left\{a_{3}\right\}$, which contains $\quad] \leftarrow, a_{2}\left[\right.$ or $a_{0}$ is a subset of $] \leftarrow, a_{1}\left[\right.$, whenever $\mathrm{a}_{3}<\mathrm{a}_{1}$, and it contains ]$\leftarrow, a_{1}\left[\right.$, whenever $a_{1}<a_{3}$. So, by definition $1, A$ is a segment.

2) Let $\overrightarrow{\mathrm{A}}$ be an oriented segment. We prove, that the order, induced by its connectedness satisfies all the conditions stated in the theorem.

a) First, we show, that a subset $\mathrm{C} \subset \overrightarrow{\mathrm{A}}$ is disconnected, if there are points $\mathrm{a}_{1}<\mathrm{a}_{2}$ $<\mathrm{a}_{3}$ in $\overrightarrow{\mathrm{A}}$, such that $\mathrm{a}_{1}, \mathrm{a}_{3} \in \mathrm{C}$, but $\mathrm{a}_{2} \notin \mathrm{C}$. By corollary 5 , one of the sets ]$\left.\leftarrow, a_{2}[;] \leftarrow, a_{2}\right]$ is open, while the other one is a closed subset of $\vec{A}$. Thus, ]$\leftarrow, a_{2}\left[\right.$ is a clopen subset of $A-\left\{a_{2}\right\}$. So, ] $\leftarrow, a_{2}[\cap C$ is a nontrivial clopen subset of $\mathrm{C}$; therefore, $\mathrm{C}$ is a disconnected subset of $\overrightarrow{\mathrm{A}}$.

b) Now we show, that subset $\mathrm{C}$ of $\overrightarrow{\mathrm{A}}$ is connected, whenever it contains together with any two of its points also all the points between them. Assuming the contrary, we get the following: there is a nontrivial clopen subset $\mathrm{U}$ of $\mathrm{C}$. There are two possibilities:

i) There are points $a_{1}<a_{2}<a_{3}$ in $C$, such that $a_{1}, a_{3}$ belong to one of the sets $\mathrm{U}, \mathrm{C}-\mathrm{U}$ and $\mathrm{a}_{2}$ belongs to the other;

ii) Each one of the sets U, C - U contains together with any two of its points also all the points between them.

In case $i)$, without loss of generality, we can assume, that $\mathrm{a}_{1}, \mathrm{a}_{3} \in(\mathrm{C}-\mathrm{U})$ and $a_{2} \in U$. By corollary 5 , one of two sets $] a_{1}, \rightarrow\left[,\left[a_{1}, \rightarrow\left[(] \leftarrow, a_{3}[\right.\right.\right.$,

]$\left.\left.\leftarrow, a_{3}\right]\right)$ is an open and the other one is a closed subset of $\vec{A}$. Thus, one of the sets $\left.] a_{1}, a_{3}[;] a_{1}, a_{3}\right] ;\left[a_{1}, a_{3}\left[;\left[a_{1}, a_{3}\right]\right.\right.$ is an open and one is a closed subset of $\overrightarrow{\mathrm{A}}$. Without loss of generality, we can assume, that set ] $\mathrm{a}_{1}, \mathrm{a}_{3}[$ is an open, and $\left[a_{1}, a_{3}\right]$ is a closed subset of $\vec{A}$. Because $a_{1}, a_{3} \notin U$, we have ] $\mathrm{a}_{1}, \mathrm{a}_{3}\left[\cap \mathrm{U}=\left[\mathrm{a}_{1}, \mathrm{a}_{3}\right] \cap \mathrm{U}\right.$. Because $\left[\mathrm{a}_{1}, \mathrm{a}_{3}\right] \subset \mathrm{C}$, and $\mathrm{U}$ is a clopen subset of $C, U \cap] a_{1}, a_{3}\left[\right.$ is an open subset of ] $a_{1}, a_{3}[$. So, the set $U \cap] a_{1}, a_{3}[$ is an open subset of $\vec{A}$, as an open subset of an open subset ] $a_{1}$, $a_{3}[$ of $\vec{A}$. Similarly, from the facts, that $\left[a_{1}, a_{3}\right] \cap U$ is a closed subset of a closed subset $\left[a_{1}, a_{3}\right]$ of $\vec{A}$, it follows, that $\left[a_{1}, a_{3}\right] \cap U$ is a closed subset of $A$. Thus, keeping in mind the equality $] a_{1}, a_{2}\left[\cap U=\left[a_{1}, a_{2}\right] \cap U\right.$, we conclude, that ] $\mathrm{a}_{1}, \mathrm{a}_{2}[\cap \mathrm{U}$ is a clopen subset of $\overrightarrow{\mathrm{A}}$. This contradicts the connectedness of $\overrightarrow{\mathrm{A}}$. So, $\mathrm{C}$ is a connected subset of $\overrightarrow{\mathrm{A}}$.

In case ii), by corollary 6 , we can conclude, that at least one of the sets $\mathrm{U}, \mathrm{C}-\mathrm{U}$ has an end-element in $\mathrm{C}$, which we denote by a. Because one of the sets $\mathrm{U}, \mathrm{C}$ $\mathrm{U}$ is a clopen subset of $\mathrm{C}$, by theorem 8 , it follows, that $\{\mathrm{a}\}$ is a clopen subset of $C$. Thus, there are such an open subset $W$ of $\vec{A}$ and a closed subset V of $\vec{A}$, 
that $\{\mathrm{a}\}=\mathrm{W} \cap \mathrm{C}=\mathrm{V} \cap \mathrm{C}$. Because $\mathrm{a}$ is an end-element of one of the sets $\mathrm{U}$, $\mathrm{C}-\mathrm{U}$ in $\mathrm{C}$, there is an interval of one of the types [ b,a [ or ] a,b ] in $\mathrm{C}$, intersection of which with one of the sets $\mathrm{U}, \mathrm{C}-\mathrm{U}$ is empty. Let us assume, that the interval is $] \mathrm{a}, \mathrm{b}] \subset \mathrm{C}$. From equality $\{\mathrm{a}\}=\mathrm{W} \cap \mathrm{C}=\mathrm{V} \cap \mathrm{C}$ it follows, that $\mathrm{W} \cap] \mathrm{a}, \mathrm{b}]=\mathrm{V} \cap] \mathrm{a}, \mathrm{b}]=\varnothing$ (here ] a,b ] is an interval of set $\mathrm{A})$. Thus, $\mathrm{a}$ is an end-element of each of the sets $\mathrm{W}, \mathrm{V}$ in $\mathrm{A}$. By theorem $8,\{\mathrm{a}\}$ is a clopen subset of $\overrightarrow{\mathrm{A}}$, what contradicts the connectedness of $\overrightarrow{\mathrm{A}}$. So, in all logically possible cases $\mathrm{C}$ is a connected subset of $\overrightarrow{\mathrm{A}}$.

Theorem 10. Subset B of a segment $\vec{A}$ is a segment itself iff $B$ is a connected subset of $\vec{A}$. Proof follows immediately from theorem 9.

\section{INTERMEDIATE VALUE THEOREM}

Let $\mathrm{f}: \mathrm{X} \rightarrow \mathrm{Y}$ is a function from a connected ordered space $\mathrm{X}$ into a connected ordered space $Y$. Let $f(a) \neq f(b)$, then for any $z \in Y$ such that $f(a)<z<f(b)$, there is a point $c \in$ $\mathrm{X}$, such that $\mathrm{a}<\mathrm{c}<\mathrm{b}$ and $\mathrm{f}(\mathrm{c})=\mathrm{z}$.

Proof follows immediately from the fact, that a continuous image of a connected set is connected, and from theorem 10.

Theorem 11. In order that an ordered set $\mathrm{C}$ admit an O-topology it is necessary and sufficient that $\mathrm{C}$ does not contain gaps and there does not exist a decomposition of the set $\mathrm{C}$, satisfying the conditions of theorem 7 .

Proof. The necessity follows from theorem 7. A sufficient condition is given in [16], theorem 6 .

\section{INTERVAL ALTERNATING TOPOLOGY}

Definition 8 . Let $\mathrm{C}$ be an ordered set. For every a $\in \mathrm{C}$, there are only two possibilities:

I. $\mathrm{a}$ is a limit element of $\mathrm{C}$;

II. $\mathrm{a}$ is an isolated element of $\mathrm{C}$.

We decompose the set of all isolated elements of $C$ into equivalence classes, as follows. Any two points belong to the same equivalence class, if there is no limit element between them. Let us choose a representative a from each equivalence class. For a, there are only the following possibilities:

1) there exists a limit element a', such that the number of elements between a and a' is finite;

2) there exists a sequence $\ldots<a_{-n}<\ldots<a_{-2}<a_{-1}=a=a_{1}<a_{2}<\ldots<a_{n}<\ldots$, such that $] a_{i}, a_{j}\left[=U a_{k}\right.$, where $\mathrm{i}<\mathrm{k}<\mathrm{j}(n=1,2, \ldots<w)$;

3) there exists a sequence $\mathrm{a}_{-\mathrm{m}}<\mathrm{a}_{-\mathrm{m}+1}<\ldots \mathrm{a}_{-2}<\mathrm{a}_{-1}=\mathrm{a}=\mathrm{a}_{1}<\mathrm{a}_{2}<\ldots<\mathrm{a}_{\mathrm{n}}<\ldots$, such that 


$$
] \leftarrow, a\left[=\bigcup_{i=1}^{m} a_{-i} \text { and }\right] a_{i}, a_{j}\left[=U a_{k} \text {, where } \mathrm{i}<\mathrm{k}<\mathrm{j}(\mathrm{n}=1,2, \ldots,<w)\right. \text {; }
$$

4) there exists a sequence $\ldots a_{-n}<:<a_{-2}<a_{-1}=a=a_{1}<\ldots<a_{m}$, such, that

$$
\left[\mathrm{a}, \rightarrow\left[=\cup_{\mathrm{i}=1}^{\mathrm{m}} \mathrm{a}_{\mathrm{i}} \text { and }\right] \mathrm{a}_{\mathrm{i}}, \mathrm{a}_{\mathrm{j}}\left[=\mathrm{Ua}_{\mathrm{k}} \text {, where } \mathrm{i}<\mathrm{k}<\mathrm{j}(\mathrm{n}=1,2, \ldots,<\mathrm{w})\right. \text {; }\right.
$$

5) there exists a sequence $\mathrm{a}_{-\mathrm{m}}<\ldots<\mathrm{a}_{-1}=\mathrm{a}=\mathrm{a}_{1}<\mathrm{a}_{2}<\ldots<\mathrm{a}_{\mathrm{n}}$, such that $\mathrm{C}=\mathrm{Ua}_{\mathrm{i}}$, where $\mathrm{m} \leq \mathrm{i} \leq \mathrm{n}$.

We introduce a topology on $\mathrm{C}$ by the following way:

We take the following sets as open sets in $\mathrm{C}$ :

] $\leftarrow$, a [ and ] a, $\rightarrow$ [ in case I and in case 1), if the number of elements between a and a' is odd;

] $\leftarrow$, a ] and [ $\mathrm{a}, \rightarrow$ [ in case 1 ) if the number of elements between a and $\mathrm{a}$ is even;

in cases 2) - 5), either sets of type $] \leftarrow, a_{ \pm}(2 k-1)[$ and $] a_{ \pm(2 k-1)} \rightarrow[$

$(\mathrm{k}=1,2, \ldots)$, or sets of type $] \leftarrow, \mathrm{a}_{ \pm 2 \mathrm{k}}[$ and $] \mathrm{a}_{ \pm 2 \mathrm{k}}, \rightarrow[$.

The open sets described above we consider as constituting a subbase of the topology, which we shall call interval alternating (i. a. ) topology.

Theorem 12. An interval alternating (i.a.) topology on an ordered set A, which does not satisfy the conditions of theorem 7, is an O-topology on A; in other words, an ordered set A, which does not satisfy the conditions of theorem 7, with i.a. topology, is a segment $\overrightarrow{\mathrm{A}}$.

Proof follows from definition 8 and theorem 9.

Corollary 11. Each i.a. topology is uniquely determined by the set of all one-point open sets in this topology.

Proof. Indeed, the subbase of an i.a. topology is a union of sets of types ] $\leftarrow$, a ] and $[\mathrm{a}, \rightarrow[$, where $\{\mathrm{a}\}$ is the set of all open one point sets, with set of sets of types ] $\leftarrow$, a [ and $[\mathrm{a}, \rightarrow[$, where $\mathrm{a}$ is a limit element.

Corollary 12. For each O-Topology $\mathrm{T}$ in an ordered set $\mathrm{C}$, there exists a uniquely determined i.a. topology, which is smaller, than $\mathrm{T}$, except in the case, when $\mathrm{C}$ is an antidiscrete two points space.

Proof: By theorems 1 and 6, it follows, that $\{a\}$ is either an open or a closed subset of $\overrightarrow{\mathrm{C}}$, where $\overrightarrow{\mathrm{C}}$ is a segment different from antidiscrete two points space and a $\in \overrightarrow{\mathrm{C}}$. If $\{\mathrm{a}\}$ is a closed (open) subset of $\overrightarrow{\mathrm{C}}$, then $] \mathrm{a}, \rightarrow[([\mathrm{a}, \rightarrow[\mathrm{)}$ and $] \leftarrow$, a $[(] \leftarrow$, a $])$ are open subsets of $\overrightarrow{\mathrm{C}}$. The collection of such open subsets of $\overrightarrow{\mathrm{C}}$ forms a subbase of $i$. a. topology on $\overrightarrow{\mathrm{C}}$, which is smaller than $\mathrm{T}$. If a is a limit point of ordered set $\mathrm{C}$ or there is an odd number of points between a and some limit point of $C$, then $\{a\}$ is a closed subset of $\vec{C}$, if there is an even number of points between a and some limit point of $\vec{C}$, then $\{a\}$ is an open 
subset of $\vec{C}$. Otherwise, we decompose the set of all those elements, that do not satisfy previous conditions, into equivalence classes by following way:

Two points $\mathrm{a}, \mathrm{b} \in \mathrm{C}$ are equivalent if ] $\mathrm{a}, \mathrm{b}$ [ is a finite set. Let $\mathrm{a}$ be a representative of such class. By theorems 1 and $6,\{a\}$ is either an open, or a closed subset of $\vec{C}$; all the points from the same equivalence class such, that there are an odd number of points between each of them and a, are open (closed) subsets of $\overrightarrow{\mathrm{C}}$, and all other points from the same class are closed (open) subsets of $\overrightarrow{\mathrm{C}}$. By previous corollary, the i.a. topology, which is smaller than $\mathrm{T}$, is unique.

Definition 9. By N. Bourbaki [1], space $\mathrm{T}$ is a quasi-compact, iff any open cover of $\mathrm{T}$ contains finite subcover.

Theorem 13. The segment (ordered topological space) [ $\overrightarrow{a, b}]$ with i.a. topology and only such segment is a quasi-compact space.

Proof: Let $\overrightarrow{\mathrm{C}}=[\overrightarrow{\mathrm{a}, \mathrm{b}}]$ be a segment with two end points and with i.a. topology. Every cover of $[\overrightarrow{a, b}]$ with elements of subbase, described in definition 8 , contains a finite subcover, containing at most two elements. Thus, by Alexander's Subbase Theorem in J. Kelly [2] or in T. Gamelin and R. Greene [25], [ $\overrightarrow{a, b}]$ is a quasi-compact space. If there is no the last end-point in the segment $\overrightarrow{\mathrm{C}}$, then $\overrightarrow{\mathrm{C}}$ is not a quasicompact space. Indeed, for each point $\mathrm{a} \in \overrightarrow{\mathrm{C}}$ one of the sets of types ] $\leftarrow$, a [ and ] $\leftarrow$, a ] is open in $\overrightarrow{\mathrm{C}}$; family of such open subsets of $\overrightarrow{\mathrm{C}}$ constitutes an open cover of $\overrightarrow{\mathrm{C}}$, which does not contain a finite subcover, because the last element of $\vec{C}$ does not exist. So, by definition, such $\vec{C}$ is not quasi-compact. If there is no the first element in $\overrightarrow{\mathrm{C}}$, similar prove can be used, by changing the order in $\overrightarrow{\mathrm{C}}$ on opposite.

Let $\vec{C}$ be a segment with a larger topology, than i.a. topology. This means, that there is an open set $U$ in $\vec{C}$ and a point $a_{0} \in U$, such that $\left\{a_{0}\right\}$ is a closed subset of $\vec{C}$, $a_{0}$ is a limit element of ordered set $\mathrm{C}$, that satisfies at least one of the following conditions:

1) for each interval of the type ] $a_{1}, a_{0}$ [ the following inequalities are true: $] a_{1}, a_{0}[\cap U$ $\neq \varnothing$ and $] a_{1}, a_{0}[\cap(\vec{C}-U) \neq \varnothing$;

2) for each interval of the type $] a_{0}, a_{1}[$ the following inequalities are true: $] a_{0}, a_{1}[\cap U$ $\neq \varnothing$ and $] a_{0}, a_{1}[\cap(\vec{C}-U) \neq \varnothing$.

In case 1) for each $a<a_{0}$ we take one of the sets ] $\leftarrow$, a [ and ] $\leftarrow$, a ], that is open in $\overrightarrow{\mathrm{C}}$, and also sets $\mathrm{U}$ and $] \mathrm{a}_{0}, \rightarrow[$. The family of such sets gives an open cover of $\overrightarrow{\mathrm{C}}$, which does not contain a finite subcover. So, $\overrightarrow{\mathrm{C}}$ is not a quasi-compact space. In case 2 ) the proof is reduced to previous by changing the order in $\overrightarrow{\mathrm{C}}$ on opposite. 
Corollary 13. If there exists a quasi-compact O-topology on ordered set $\mathrm{C}$, then such topology is not unique in general, and any two such topologies are not comparable.

Proof. By definition 8, the i.a. topology on ordered set $\mathrm{C}$ is not unique in general, if it exists. By the proof of theorem 13, any O-topology on $\mathrm{C}$, which is larger, than some i.a. topology, is not a quasi-compact topology; and, by corollary 12, neither one O-topology different from some i.a. topology, is an i.a. topology.

Theorem 14. If all cross-sections in ordered set $\mathrm{C}$ are jumps, then all O-topologies in $\mathrm{C}$ are i.a. topologies.

Proof. By theorem 11, an O-topology on C exists. If every cross-section in $\mathrm{C}$ is a jump, then $\mathrm{C}$ permits exactly one of the following representations:

1) $\mathrm{C}=\left\{\ldots<\mathrm{a}_{-\mathrm{m}}<\ldots \mathrm{a}_{-1}<\mathrm{a}_{0}<\mathrm{a}_{1}<\mathrm{a}_{2}<\ldots \mathrm{a}_{\mathrm{m}}<\ldots\right\}$, where $\mathrm{m}=1,2, \ldots,<\mathrm{w}$;

2) $\mathrm{C}=\left\{\mathrm{a}_{1}<\mathrm{a}_{2}<\ldots<\mathrm{a}_{\mathrm{m}}<\ldots\right\}$, where $\mathrm{m}=1,2, \ldots, \mathrm{w}$;

3) $\mathrm{C}=\left\{\ldots<\mathrm{a}_{-\mathrm{m}}<\ldots<\mathrm{a}_{-2}<\mathrm{a}_{-1}\right\}$, where $\mathrm{m}=1,2, \ldots,<\mathrm{w}$;

4) $\mathrm{C}=\left\{\mathrm{a}_{1}<\mathrm{a}_{2}<\ldots<\mathrm{a}_{\mathrm{k}}\right\}$, where $\mathrm{k}$ is a natural number.

Let $\mathrm{T}$ be an arbitrary O-topology on $\mathrm{C}$, and $\mathrm{B}$ is a connected subset of $\overrightarrow{\mathrm{C}}$ with topology T. By definition of O-topology, ordered set $\mathrm{C}$ with topology $\mathrm{T}$ is a segment $\overrightarrow{\mathrm{C}}$. By theorem 9 , and because every cross-section of $\mathrm{C}$ is a jump, B permits exactly one of the following representations:

a) $\mathrm{B}=\mathrm{C}$;

b) $\mathrm{B}=[\mathrm{a}, \rightarrow[$;

c) $\mathrm{B}=] \leftarrow, \mathrm{a}]$;

d) $B=[a, b]$; where $a, b \in \vec{C}$, and neither of them is an endpoint of $\vec{C}$. Each one of the points $a, b$ is an end-element of $B$ in $\vec{C}$. Let $U$ be an open subset of $\vec{C}$ and $B$ be $a$ component of $U$. Each one of the points $a, b$ is an end-element of $U$ in $\vec{C}$. So, by theorem $8,\{a\}$ and $\{b\}$ are open subsets of $\vec{C}$. Let $P$ is an i.a. topology on $\vec{C}$, which is smaller, than $T$. Existance of $P$ follows from corollary 12. The sets $\{a\},\{b\}$ are open subsets of $\overrightarrow{\mathrm{C}}$ with topology $\mathrm{P}$, so, by definition of i.a. topology, set $\mathrm{B}$ is open under $\mathrm{P}$. Thus, $U$ is also open under topology $P$. Because $U$ is an arbitrary element of $T(U \in T)$ and $\mathrm{P} \subseteq \mathrm{T}$, it follows, that $\mathrm{P}=\mathrm{T}$. So, every $\mathrm{O}$-topology on $\mathrm{C}$ is an i.a. topology.

Definition 10. Let $\mathrm{S}$ be an infinite sequence

$$
\ldots<a_{-n}<a_{-n+1}<\ldots<a_{-2}<a_{-1}<a_{0}<a_{1}<a_{2}<\ldots<a_{n}<\ldots
$$

where $\mathrm{n}$ is an arbitrary integer number. By theorem 12, ordered set $\mathrm{S}$ with i.a. topology on it is an ordered topological space, and one of orders, induced by its connectedness coincides with the order of sequence $S$. Such a segment $\vec{S}$ we shall call a sequence-line.

Corillary 14. All O-topologies on sequence $\mathrm{S}$ are i.a. topologies ( $\mathrm{S}$ is a sequence-line). So sequence $\mathrm{S}$ with arbitrary O-topology is a sequence-line.

Proof follows from theorem 14. 
Corollary 15. There are exactly two O-topologies on sequence-line and they are noncomparable.

Definition 11. An integer line is the set of all integers with its natural order and with i.a. topology. A digital line is a finite connected subset of the integer line.

Corollary 16. Any integer line is a connected ordered topological space.

Proof follows immediately from definition 10 .

Corollary 17. Any digital line is a connected ordered topological space (segment).

Proof follows from theorem 10.

Corollary 18. Every digital line is a quasi-compact space.

Proof follows from theorem 13.

Definition 12. An integer (digital) arc is a homeomorphic image of integer (digital) line.

Corollary 19. Any integer arc is a sequence-line.

Corollary 20. Any digital arc is a finite sequence of points $a_{1}, a_{2}, \ldots, a_{n}$ with i.a. topology.

Definition 13. A digital curve is a factor space of digital arc with odd number of points greater, than 3 , obtained by identification of its two ends.

\section{DIGITAL ANALOG OF INTERMEDIATE VALUE THEOREM}

Theorem 15. Let $\mathrm{X}$ and $\mathrm{Y}$ be two digital or integer arcs and $\mathrm{f}: \mathrm{X} \rightarrow \mathrm{Y}$ is a continuous function such, that $f(a) \neq f(b)$. Then for any $z \in Y$ and such, that $f(a)<z<f(b)$, there is a point $\mathrm{c} \in \mathrm{X}$ such, that $\mathrm{a}<\mathrm{c}<\mathrm{b}$ and $\mathrm{f}(\mathrm{c})=\mathrm{z}$.

Proof follows from general intermediate value theorem.

Definition 14. A path (Digital path) is a continuous image of connected ordered topological space (digital arc).

Definition 15. A topological plane or picture (integer, digital plane) is a topological product of two segments (integer, digital lines) correspondingly. $\mathrm{N}$-dimensional topological space $\mathrm{S}^{\mathrm{N}}$ or Hilbert space $\mathrm{H}$ (integer, digital space) is, correspondingly, a topological product of $\mathrm{N}$ or countably many segments (integer, digital lines).

Definition 16. A topological (digital) $\mathrm{N}$-cube $\mathrm{D}^{\mathrm{N}}$ is a topological product of $\mathrm{N}$ copies of a segment with two end points (digital lines).

Topology of digital lines and plane is described in [ 20-24 ]. 


\section{DIGITAL CUBES DN , N-DIMENSIONAL DIGITAL SPACES}

Let $\left[\overrightarrow{a_{0}, a_{n}}\right]=a_{0}<a_{1}<a_{2}<\ldots<a_{n}$ be a digital line, and all the points of the type $a_{2 k}(0 \leq 2 k \leq n)$ are open subsets of $\left[\overrightarrow{a_{0}, a_{n}}\right]$. By corollary 11 , this uniquely describes the i.a. topology on $\left[\overrightarrow{a_{0}, a_{n}}\right]$. Every point of $D^{N}=\left[\overrightarrow{a_{0}, a_{n}}\right]^{N}$ with $N$ coordinates $\left(a_{i_{1}}, a_{i_{2}}, \cdots, a_{i_{N}}\right)$, all with even indeces, is an open subset of $D^{N}$; every point $D^{N}$ with $N$ coordinates and odd indeces is a closed subset of $\mathrm{D}^{\mathrm{N}}$; all other points are neither open, nor closed subsets of $\mathrm{D}^{\mathrm{N}}$ and are called neutral. By definition of product topology, one can easily describe sets of open and closed subsets of $\mathrm{D}^{\mathrm{N}}$.

Definition 17. A space $\mathrm{X}$ is path connected if for any two points $\mathrm{x}, \mathrm{y} \in \mathrm{X}$ there is a path $f:[\overrightarrow{a, b}] \rightarrow X$, such that $f(a)=x, f(b)=y$.

Definition 18. A space $\mathrm{X}$ is locally pathconnected, if for any point $\mathrm{x} \in \mathrm{X}$ and its neighborhood $\mathrm{U}(\mathrm{x})$ there is an open partconnected neighborhood $\mathrm{V}(\mathrm{x}) \subset \mathrm{U}(\mathrm{x})$.

Theorem 16. A topological N-Dimensional space, which is a product of $\mathrm{N}$ segments with i.a. topology is a locally path-connected topological space.

Proof follows from definitions of i.a. topology and a product topology.

Corollary 21. Any digital (integer) N-dimensional space is locally pathconnected.

Theorem 17. Connectedness and pathconnectedness are equivalent in $\mathrm{N}$-dimensional spaces described in theorem 16.

Proof follows from theorem 16 and from connectedness of the spaces.

Corollary 22. Connectedness and pathconnectedness in digital (integer) $\mathrm{N}$-spaces are equivalent.

The results presented here is the first topological formalism of spaces with at most countably many points. They also show, how to apply the methods of continuous mathematics to areas, that for a long time have been considered to be under the total control of discrete mathematics.

\section{REFERENCES}

1. Bourbaki, N. Topologie General, Hermann, Paris, [1953].

2. Kelley, J. General Topology, Van Nostrand, New York, [1955]; Springer-Verlag, [1975]. 
3. Spanier, E. Algebraic Topology, McGraw-Hill, [1966].

4. Hu, S. Homotopy Theory, Academic Press, [1959].

5. Greenberg, M. Lectures on Algebraic Topology, Benjamin, New York, [1967].

6. Rosenfeld, A. Arcs and curves in digital pictures, J. Assoc. Comput. Mach 20:81-87 [1973].

7. . Digital Topology, AM Monthly 86(8):621-630 [1979].

8. $\overline{[1970]}$. Connectivity in digital pictures, J. Assoc. Comput. Mach. 17:146-160

9. . Picture Languages, Academic Press, New York, [1979].

10. Udura, J., S. Srihari, G. Herman. Boundary detection in multidimensions, IEEE Trans. Pattern Anal, and Mach. Intell. 4(1):41-62 [1982]

11. Minsky, M. and S. Papert. Perceptrons: An introduction to comp. geom., MIT Press, Cambridge, Mass. [1969].

12. Sklansky, J. Recognition of convex blobs, Pattern recognition 2:3-10 [1970].

13. Hurewicz, W. and H. Wallman. Dimension theory, Princeton U. Press, Princeton, NJ, [1941].

14. Mylopoulos, J. and T. Pavlidis. On the topological properties of quantized spaces. I. The notion of dimension, J. ACM 18(2):239-246 [1971].

15. . II. Connectivity and order connectivity, J.ACM 18(2):247-254 [1971].

16. Khalimsky, E. On topologies of generalized segments. Sov. Math. Docl. 10(6):15081511 [1969].

17. The generalized segment and its use in homotopy theory, Sov. Math. Docl.

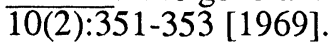

18. Ordered topological spaces and their applications, Dissertation, Moskow Pedag, Institute, [1969].

19. _ . Ordered Topological Spaces, Naukova Dumka Press, Kiev, [1977].

20. . Pattern analysis of N-dimensional digital images, Proc. IEEE Conf. Systems, man, and cybernetics, Atlanta, GA, 1559-1562 [1986].

21. Digital image processing and topology, Proc. Conf. Comp. \& Math., Palo Alto, CA, [1986].

22. . Digital picture processing, Proc. SIAM meeting, Syst. \& Signals, Boston [1986]. 
23. . Topological foundation of pattern recognition in computer and robot vision, Proc. First Intern. Congr. on Comp. Vision, London [1987].

24. . Topological methods in computer science, Intern. Congress of Mathematicians, Berkeley, CA, [1986].

25. Gamelin, T., R. Green. Intro to Topology, Saunders, NY, [1983]. 


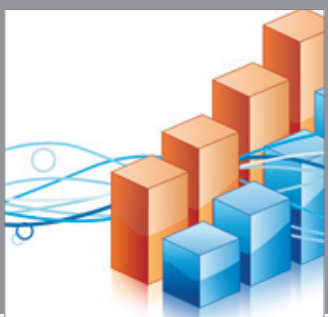

Advances in

Operations Research

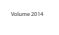

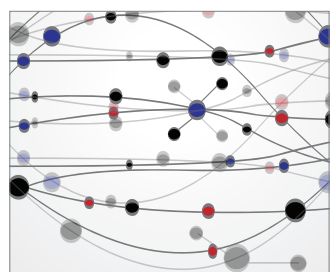

\section{The Scientific} World Journal
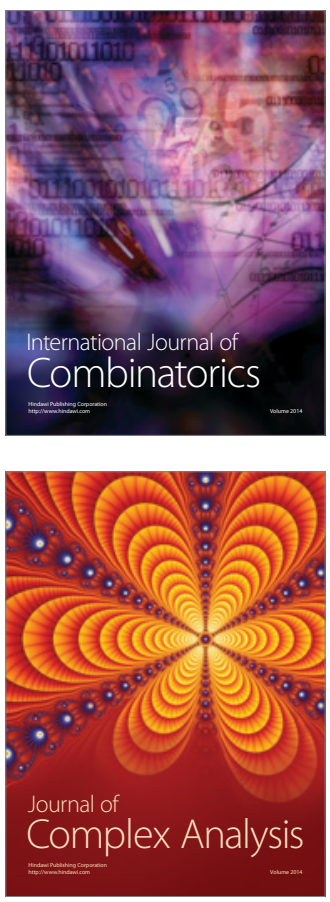

International Journal of

Mathematics and

Mathematical

Sciences
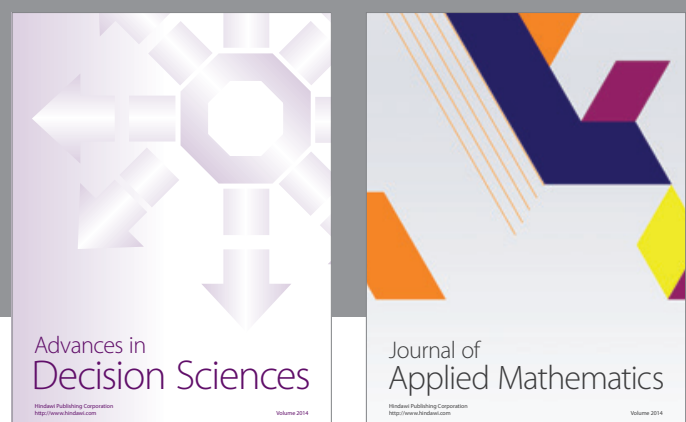

Journal of

Applied Mathematics
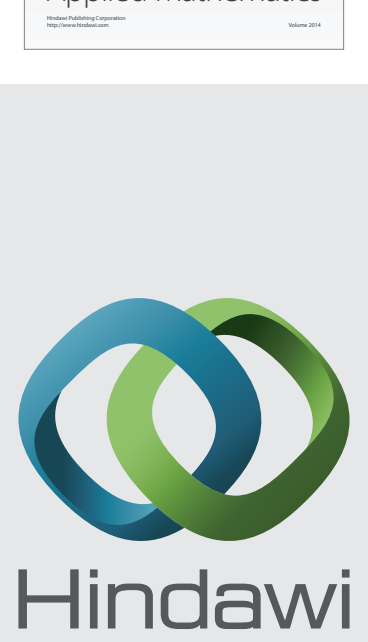

Submit your manuscripts at http://www.hindawi.com
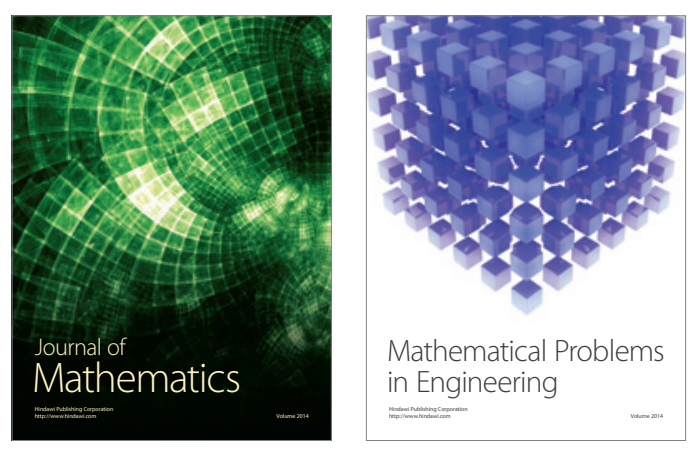

Mathematical Problems in Engineering
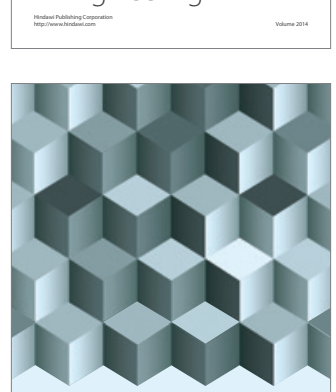

Journal of

Function Spaces
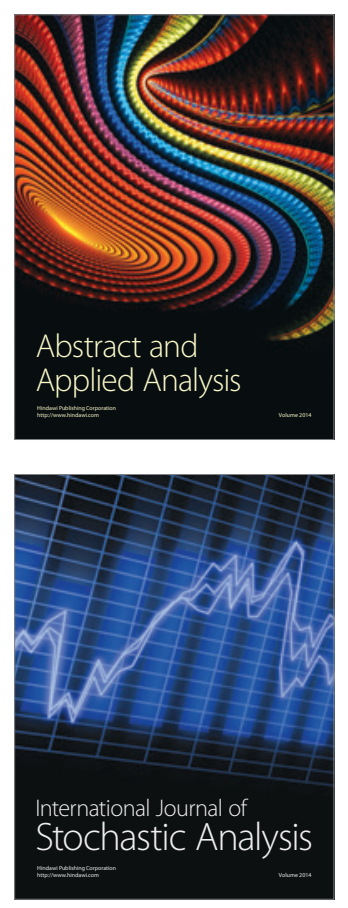

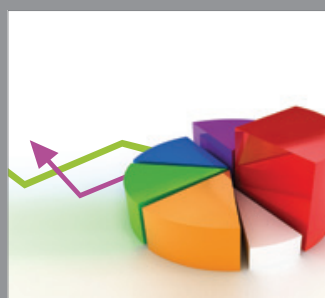

ournal of

Probability and Statistics

Promensencen
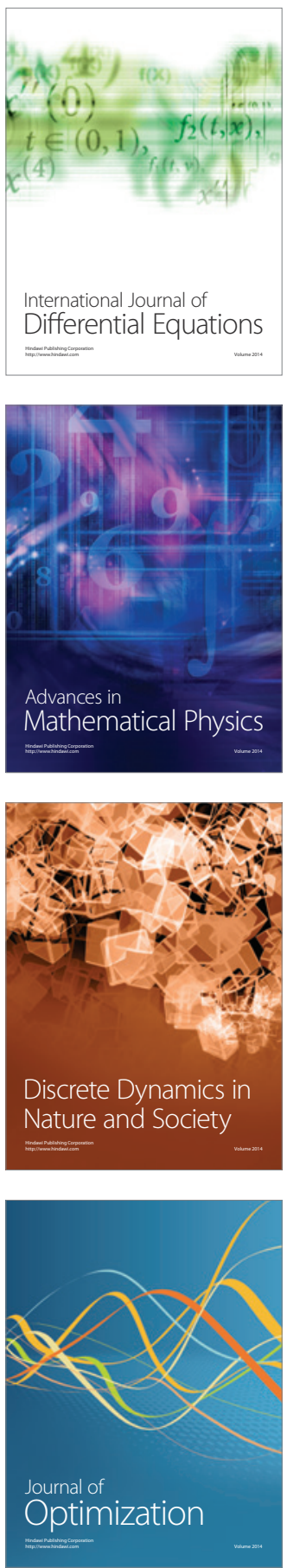